\title{
THE LOCALIZATION OF ANEURIN PYROPHOSPHATASE IN THE BRAIN
}

\author{
BY \\ D. NAIDOO and O. E. PRATT \\ From the Departments of Neuropathology and Biochemistry, Institute of Psychiatry, \\ Maudsley Hospital, University of London
}

Vitamin $B_{1}$ is nearly exclusively present in animal tissues as aneurin pyrophosphate (Ochoa and Peters, 1938). The vitamin is first converted to aneurin pyrophosphate by the tissues and in this form it enables the oxidative decarboxylation of pyruvic acid to take place allowing glucose metabolism to go beyond the pyruvate stage (Banga, Ochoa, and Peters, 1939). It has been shown more recently that aneurin pyrophosphate participates in other metabolic processes in nervous tissue (Green, Stumpf, and Zarudnaya, 1947). With regard to pyruvate oxidation, Banga and his colleagues (1939) found that free aneurin is more effective than aneurin pyrophosphate in stimulating the respiration of brain preparations from vitamin $\mathbf{B}_{\mathbf{1}}$-deficient animals. A possible explanation of this finding is that the tissue is poorly permeable to aneurin pyrophosphate but more permeable to aneurin. Aneurin consequently is able to pass the permeability barriers, become phosphorylated and be available to act as the co-enzyme. This view presupposes some dephosphorylating mechanism in the living animal whereby aneurin pyrophosphate is degraded to free aneurin.

The dephosphorylation of aneurin pyrophosphate by brain tissue was observed by Ochoa (1939). Westenbrink, Parvé, and Goudsmit (1943) described an active phosphatase hydrolysing the phosphoric esters of aneurin. It was found that this enzyme successively removes the two phosphate groups from aneurin pyrophosphate, so that when the terminal phosphate is removed, aneurin orthophosphate appears as the intermediary which is further broken down, releasing aneurin.

The histological demonstration in brain tissue of the sites of this hydrolytic process is of interest in relation to the pathology of those nervous diseases associated with aneurin deficiency.

This paper describes and illustrates the results obtained from localizing in brain tissue the sites of enzymatic cleavage of phosphate from aneurin pyrophosphate at $p \mathrm{H} 6.9$.
Method

Normal, young, adult male and virgin female white rats were used. The brains were removed rapidly under ether anaesthesia, sliced into $2 \mathrm{~mm}$. coronal sections, frozen at $-65^{\circ} \mathrm{C}$ in isopentane, and dried under vacuum. The procedure employed for the preparation of sections was in no way different from that previously described for the localization of the sites of activity of phosphatases (Naidoo and Pratt, 1951). The site of enzymatic cleavage of the phosphoric acid ester used as the substrate was demonstrated by the precipitation of the liberated phosphate as an insoluble lead salt at the site of action. The lead phosphate was converted to dark brown lead sulphide, the distribution of which was studied under the microscope.

The most suitable substrate medium for the incubation of sections was constituted thus :

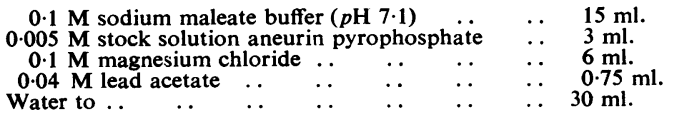

The final $p \mathrm{H}$ was between $6 \cdot 8$ and $7 \cdot 0$.

Media were prepared freshly before use. Stock solutions of substrates were neutralized with $\mathrm{NaOH}$ and stored at $-78^{\circ} \mathrm{C}$. The other constituents were either of analytical grade or were recrystallized. Glassdistilled water was used throughout. The aneurin pyrophosphate used was obtained from Roche Products (cocarboxylase), and was specified as being virtually free from other phosphorylated derivatives of aneurin. Samples used in this work were re-precipitated twice by solution in a little $\mathrm{N}-\mathrm{HCl}$ and the addition of 5 volumes of acetone. Re-precipitation did not lead to any discernible change in the histological results, nor did an independently prepared specimen for which we are indebted to Dr. A. L. Morrison of Roche Products Ltd.

The aneurin orthophosphate solution was prepared from aneurin pyrophosphate. Aneurin pyrophosphate $(100 \mathrm{mg}$.) was hydrolysed by heating in a sealed tube with $\mathrm{N}-\mathrm{HCl}\left(3 \mathrm{ml}\right.$.) for 30 minutes at $100^{\circ} \mathrm{C}$., brought to $p \mathrm{H}$ 6.8 with $\mathrm{N}-\mathrm{NaOH}$, and $\mathrm{M}-\mathrm{BaCl}_{2}$ solution $(1 \mathrm{ml}$.) added. The barium phosphate precipitate was discarded and excess barium ions removed by the addition of a slight excess of $1 \% \quad \mathrm{Na}_{2} \mathrm{SO}_{4}$ solution. The precipitate was discarded and after phosphate analysis the solution was diluted to contain $155 \mu \mathrm{g}$. of organic phosphate per ml. 
Control of the tissue activity could be obtained by omission of the phosphoric acid ester from the medium and by heating the sections in steam before incubation or by treating them with trichloracetic acid. In all these cases, and also when the magnesium salt was omitted from the medium, the staining was negligible. The intensity of staining was greatly reduced if uranyl acetate $\left(10^{-4} \mathrm{M}\right)$ was added to the medium, but sodium fluoride $\left(10^{-3} \mathrm{M}\right)$ produced no effect.

When the medium described above was used, characteristic staining was recognizable after 10 minutes' incubation, and slides were removed at intervals up to 80 minutes. If the aneurin pyrophosphate in the medium was replaced by a similar concentration of either aneurin orthophosphate or of inorganic pyrophosphate, the results were negative in each case for a comparable range of incubation times. Positive results were obtained only after incubation periods of five hours or longer. If the $p \mathrm{H}$ of the aneurin pyrophosphate medium was varied over a range from 5.0 to $8 \cdot 0$, a similar type of staining to that usually seen was observed, but the intensity reached in a given time was reduced, especially at the more acid $p \mathbf{H}$ values.

\section{Results}

The findings described represent the sites of lead sulphide deposition at the points of phosphate liberation from aneurin pyrophosphate in 10 consecutive animals.

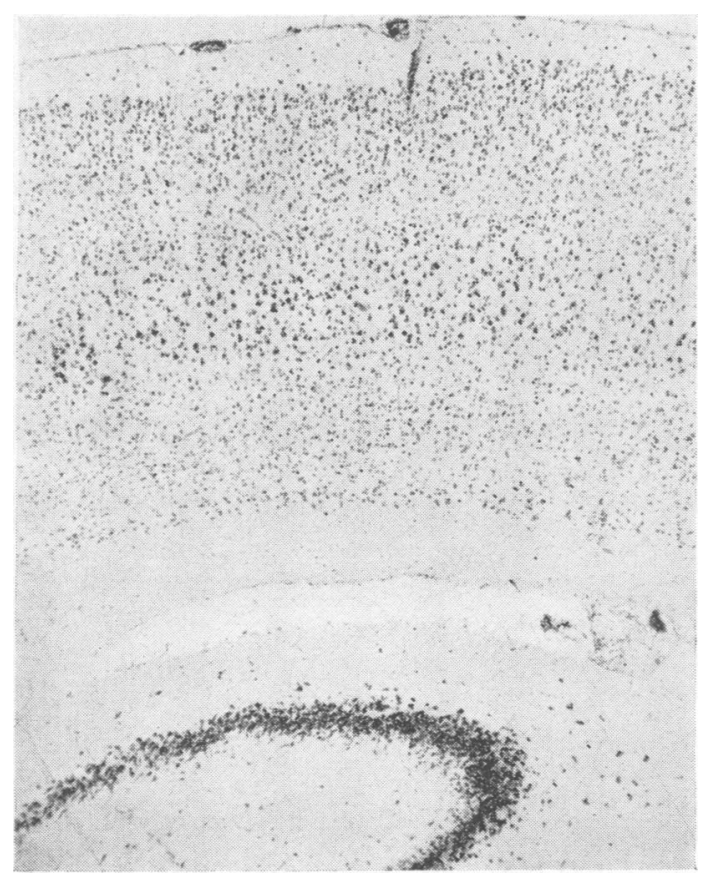

Fig. 1.-Cerebral cortex showing deep staining of the nerve cell bodies and glia. $\times 30$.
The cerebral cortex showed the characteristic cytoarchitecture. The cell bodies were clearly demarcated throughout the whole brain and frequently layers III and V of the cerebral cortex were prominent, appearing more deeply stained than the other layers. The cortex in a coronal section through the optic chiasma showed layers II and V as the most deeply stained (Fig. 1). The degree of staining of the cortical layers, however, appeared to vary from region to region. The whole cortex gave the impression of a good Nissl cresyl violet preparation with the differences in the depth of staining more than usually marked. The cell cytoplasm, nucleus, and nucleolus were stained but in uneven degree, the cytoplasm and nucleolus being more deeply stained than the nucleus. The cytoplasm did not stain evenly throughout but amorphous aggregates of deeply staining material were evident in some cells at the periphery and in others around the nucleus. It was not clear from an examination of the frozen dried material whether or not these aggregates represented Nissl bodies. The nucleus itself showed a light, more even deposit of lead sulphide but scattered throughout the nucleoplasm were small discrete granules of highly stained material. The nucleolus was consistently the darkest structure in the cortical cell body. Cells of layer V frequently showed deep staining for a short distance along their dendrites.

The nerve cell bodies in the hippocampal formation (Fig. 2), the habenula, the striatum, thalamus and hypothalamus (Fig. 3) stained deeply. The most deeply staining nerve cells in these regions were those of the hippocampus, the large cells of the globus pallidus and of the putamen (Fig. 4), and the paraventricular nucleus of the hypothalamus. The nucleus supraopticus and the mammillary nuclei stained deeply and were demonstrable after the shorter incubation periods, but their depth of staining was not as great as that of the fascia dentata of the hippocampus. The cells of the amygdaloid nuclei were consistently deeply stained.

In the midbrain, pons, and medulla nerve cells stained more lightly than the cells of the cerebral cortex, except for the nerve cells of the periaqueductal grey matter which stained deeply. Nerve cell bodies of the cranial nerve nuclei were lightly stained and were recognizable as pale brown cell bodies at an incubation time of 40 minutes.

In the cerebellar cortex the Purkinje cells and the Bergmann glia stained selectively (Fig. 5). The earliest cerebellar cells to show staining were the Bergmann glia, but after the usual incubation period of 40 minutes the Purkinje cells reached an equal depth of staining. 


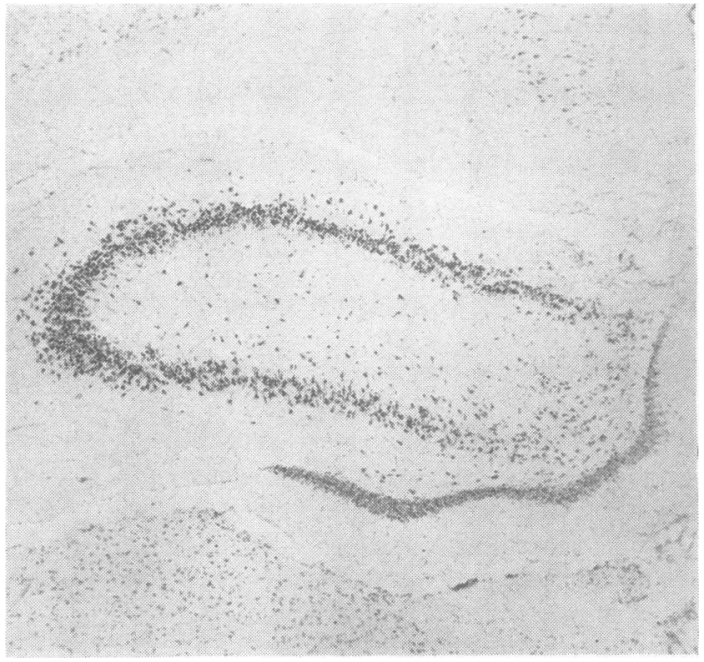

FIG. 2.-Hippocampus : cell bodies of the hippocampus (pyramidal layer and dentate gyrus) stain deeply. $\times 30$.

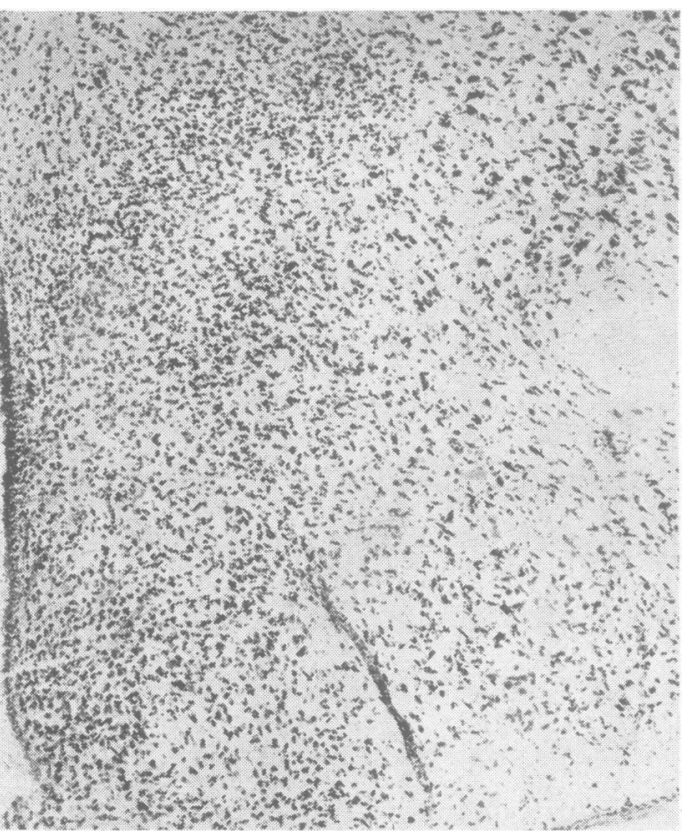

Fig. 3.-The hypothalamic nerve cells stain more deeply than those from other regions of the brain except the hippocampus. The larger intracerebral vessels show deep intimal staining. $\times 35$.

Glial cells were stained deeply throughout the whole brain. Microglia and oligodendroglia whereever seen were intensely stained, but astrocytes varied in their degree of staining even after prolonged incubation times. The endothelial nuclei

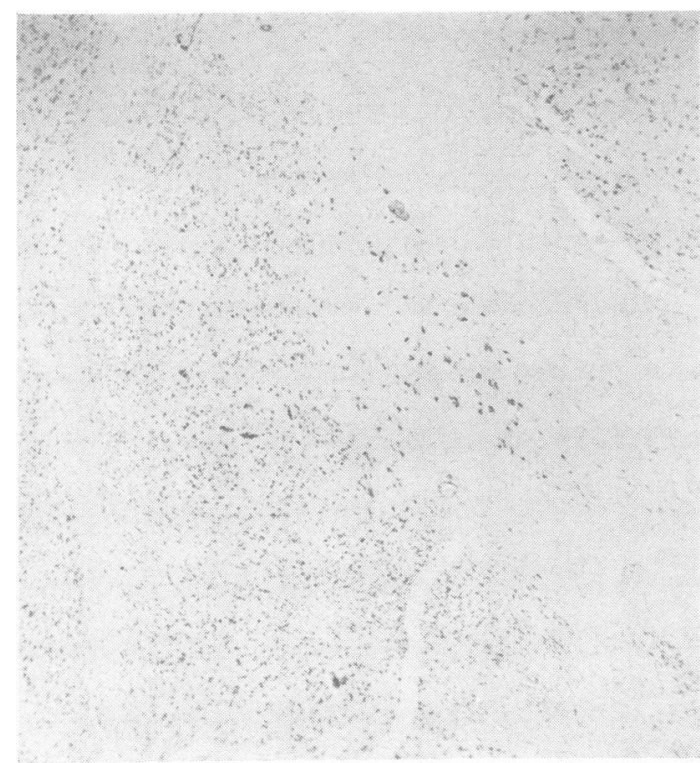

Fig. 4.-Lentiform nucleus : the large cells of the globus pallidus and putamen are prominent. $\times 30$.

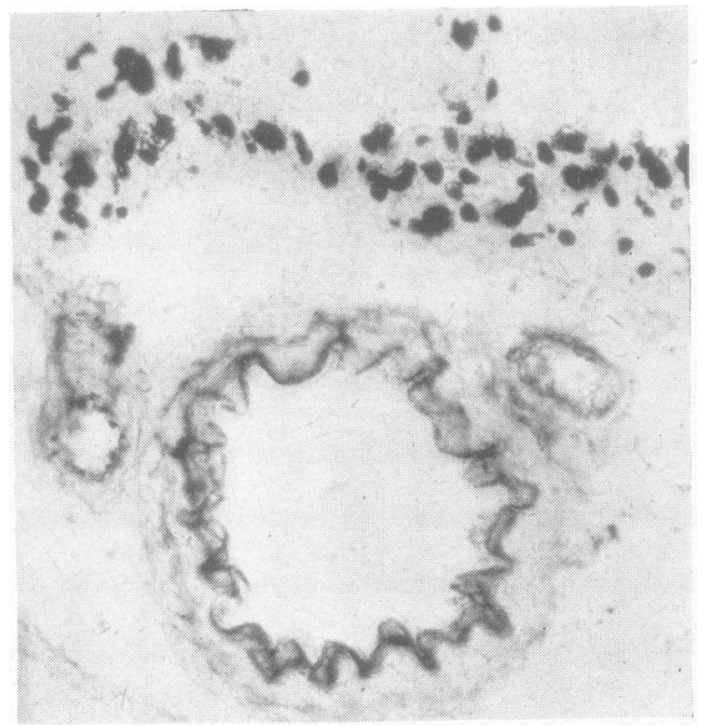

FIG. 5.-Large artery at the base of the brain showing staining of the intima and lamina elastica. $\times 100$.

of the choroidal capillaries stained moderately but the choroidal cells did not stain. Ependymal cells lining the ventricles were lightly stained. The parenchymal capillaries did not show staining but the larger intracerebral and extracerebral vessels 


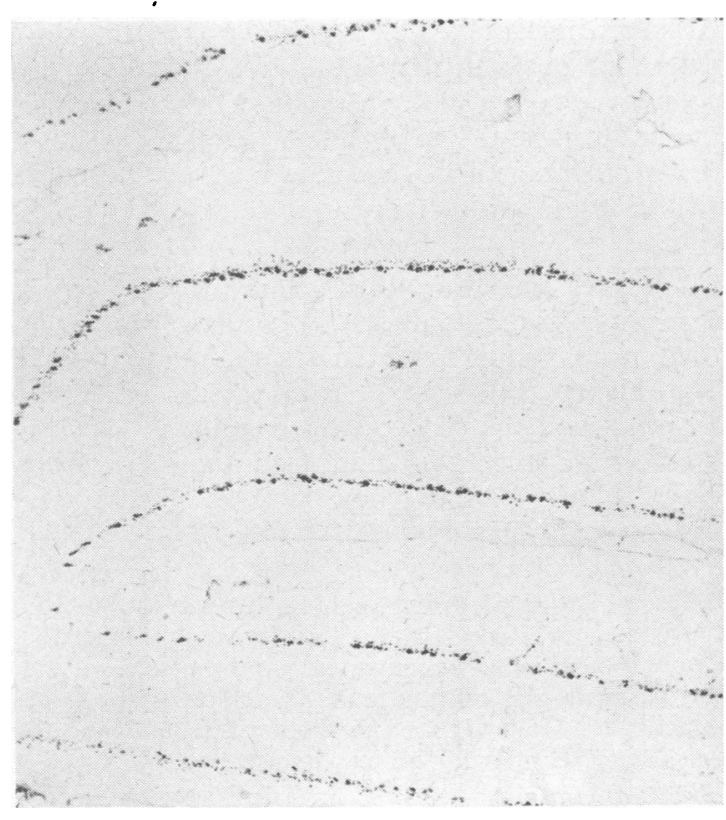

FIG. 6.-Cerebellar cortex : the Purkinje cells and Bergmann glia are the only structures which stain deeply. $\times 50$.

showed deep staining of the intima and the lamina elastica (Fig. 6). Throughout the intima of these vessels were deeply stained, small, discrete granules.

\section{Discussion}

That the localizations described represent the sites of enzyme action has been established with a fair degree of certainty by the absence of staining when aneurin pyrophosphate is omitted from the incubation medium ; by the suppression of staining when the magnesium ions are omitted, or when a low concentration of the inhibitor uranyl acetate is added ; and by heating or chemical treatment of the sections before incubation. The change seen in the localization of staining due to the replacement of the aneurin pyrophosphate by another phosphoric acid ester, such as 5-adenylic acid, provides further confirmatory evidence that enzymatic dephosphorylation of aneurin pyrophosphate has been demonstrated. It precludes the possibility of the pictures being produced to any appreciable extent by the deposition of the precipitate on foci of crystallization within the tissue (Naidoo and Pratt, 1951).

The activity detected in the present investigation appears to be distinct from that of non-specific phosphatases demonstrated by glycerophosphate. This activity has not been found in nervous tissue by previous workers who have used aneurin pyrophosphate as a substrate, presumably on account of their less favourable fixation methods.

The localizations obtained by the incubation of frozen dried brain tissue with aneurin pyrophosphate at $p \mathrm{H} 6.9$ show certain similarities to those obtained with glycerophosphate at $p \mathrm{H} 5 \cdot 3$. But there are chemical and histological differences.

Tissue incubated with aneurin pyrophosphate at $p \mathrm{H} 5 \cdot 3$ or with glycerophosphate at $p \mathrm{H} 6.9$ stains in each case at a greatly reduced rate. This difference in the $p \mathrm{H}$ optima, possibly explicable by differences in the physico-chemical properties of the two substrates, is. large enough to suggest that it is an integral part of the conditions necessary for the demonstration of separate enzyme activities. Fluoride suppresses staining in the glycerophosphate medium but has no effect on the rate of staining when aneurin pyrophosphate is used. When magnesium ions are omitted from the medium, staining is completely absent in the aneurin pyrophosphate medium but only reduced in rate when glycerophosphate is employed. It appears likely that the reaction demonstrated is the splitting of aneurin pyrophosphate to aneurin orthophosphate since replacement of the aneurin pyrophosphate in the medium by aneurin orthophosphate or by inorganic pyrophosphate fails to produce positive results within the normal incubation period.

Although there is a similarity between the picture obtained with aneurin pyrophosphate and that with glycerophosphate, some histological differences are present. In the cerebral cortex layer VI is much less prominent than in glycerophosphate material and the nuclei of neurons do stain. The cells of the cranial nerve nuclei are more lightly stained than corresponding cells in glycerophosphate sections. In the cerebellum basket fibres, which stain with material incubated with glycerophosphate, do not appear. Bergmann glia, which do not stain in sections incubated with glycerophosphate, appear first and stain most deeply of all cerebellar structures. Similar intense staining of the Bergmann glia is found when adenosine triphosphate is used as the substrate. The choroid plexus stains moderately in contrast to the deeper staining found when glycerophosphate is used. A feature which appears in the aneurin pyrophosphate material but not in glycerophosphate incubated sections is the intimal staining of the larger vessels and arterioles. The discrete and granular appearances in the intima, however, do not necessarily indicate a particulate distribution of the enzyme.

The significance of these histological and biochemical differences is not immediately apparent. 
Although it is well established that aneurin pyrophosphate functions as a co-enzyme, the mechanism of its action is not clear. The biochemical study by Westenbrink and his colleagues of the breakdown by an alkaline phosphatase of aneurin pyrophosphate in a wide range of animal tissues does not directly assist in the interpretation of the present results. Further, these authors find that aneurin orthophosphate is split readily by this phosphatase, whilst the process now studied histologically in brain appears to be concerned more directly with the hydrolysis of aneurin pyrophosphate to aneurin orthophosphate and to be distinct from the action of non-specific phosphatases. Investigation of the conditions under which aneurin orthophosphate is hydrolysed to aneurin by brain tissue sections and the demonstration of the sites of this hydrolysis would be of value.

Banga, Ochoa, and Peters (1939) find that the greater effectiveness of aneurin compared with that of aneurin pyrophosphate in stimulating the respiration of brain slices from aneurin-deficient animals can be explained by the failure of aneurin pyrophosphate to penetrate into the tissue. As aneurin is the diffusible form, at some stage dephosphorylation may be essential to the assimilation or further utilization of the vitamin. Thus sites of intense aneurin pyrophosphatase activity may indicate loci of high vitamin $\mathbf{B}_{1}$ requirements. Alternatively, the enzyme localized at these sites may function in some more complex process specific to the vitamin but of a nature not yet understood.
Although further anatomical study is necessary to determine in detail the degree of hydrolysis of the co-enzyme taking place in the various structures of the brain, it is of interest at this stage to note that the hypothalamus in general stains very deeply.

\section{Summary}

The sites of hydrolysis of aneurin pyrophosphate (cocarboxylase, vitamin $B_{1}$ pyrophosphate) have been demonstrated in frozen dried normal rat brain. The histological sites at which this activity is localized and the biochemical conditions in which it can be demonstrated differ from those previously described when other phosphoric acid esters were used as substrates.

We are indebted to the Bethlem Royal Hospital and the Maudsley Hospital, and to the Medical Research Council (Mapother Bequest) for grants made in aid of this research. We wish to thank Professor Alfred Meyer and Dr. H. Mcllwain for their interest in the work and Miss Joyce Hinton for her capable technical assistance.

\section{REFERENCES}

Banga, I., Ochoa, S., and Peters, R. A. (1939). Biochem. J., 33, 1109.

Green, D. E., Stumpf, P. K., and Zarudnaya, K. (1947). J. biol. Chem., 167, 811.

Naidoo, D., and Pratt, O. E. (1951). Journal of Neurology, Neurosurgery and Psychiatry., 14, 287.

Ochoa, S. (1939). Biochem. J., 33, 1262.

- and Peters, R. A. (1938). Ibid., 32, 1501.

Westenbrink, H. G. K., Parvé, E. P. S., and Goudsmit, J. (1943). Enzymologia, Amst., 11, 26. 